\title{
Processed 5G Signals Mathematical Models for Positioning considering a Non-Constant Propagation Channel
}

\begin{abstract}
Anne-Marie TOBIE
TéSA (ENAC)

Toulouse, FRANCE anne-

marie.tobie@ recherche.enac.fr

Abstract ranging performance of the upcoming fifth generation (5G) signal. In order to do so, it is required to define $5 \mathrm{G}$ correlator outputs mathematical models. 5G systems will use OFDM (Orthogonal Frequency Division Multiplexing) signals; in the literature, mathematical models of OFDM signals are developed at the different receiver signal processing stages. These models assumed that the propagation channel is constant over an OFDM symbol; nevertheless, an in-depth study of QuaDRiGa, a 5G compliant propagation channel simulator, invalidates this hypothesis. Therefore, in this paper, mathematical models are developed that take into account the channel evolution. The focus is given on correlator outputs and results are applied to the computation of 5G based pseudo range accuracy.
\end{abstract}

Keywords-5G systems, CIR, correlator outputs, DLL, GNSS, OFDM, propagation channel, QuaDRiGa

\section{INTRODUCTION}

Over the past few years, the need for positioning, and thus the number of positioning services in general, has been in constant growth. This need for positioning has been increasingly focused on constraint environments, such as urban or indoor environments, where GNSS (Global Navigation Satellite System) is known to have significant limitations: multipath as well as the lack of Line-of-Sight satellite visibility degrades the GNSS positioning solution and makes it unsuitable for some urban or indoor applications. In order to improve the GNSS positioning performance in constrained environments, many solutions are already available: hybridization with additional sensors or the use of signals of opportunity for example, [1], [2], [3], [5]. Concerning signals of opportunity, mobile communication signals, such as the 4G LTE ([1]) or 5G, are naturally envisioned. Indeed, a significant number of users are expected to be "connected-users" and 5G systems offers promising opportunities.

5G technology is being currently standardized and its positioning performance, as well as a potential generic receiver scheme to conduct positioning operations, is still under analysis. In order to develop such a positioning module, it is of the utmost importance to correctly characterize the mathematical models of the processed $5 \mathrm{G}$ signals at each stage of the receiver: the knowledge of these mathematical models will allow for the development of

\author{
Paul THEVENON \\ ENAC \\ Toulouse, FRANCE \\ thevenon@recherche.ena \\ c.fr
}

signal processing algorithms optimally adapted to the $5 \mathrm{G}$ received signal.

Previous efforts have been made to provide the mathematical model of the $5 \mathrm{G}$ received signal at the different receiver signal processing stages. For example, taking into account that, as defined in 3GPP standard, 5G systems use Orthogonal Frequency Division Multiplexing (OFDM) signals, the OFDM signal-type correlator output mathematical model and acquisition techniques derived in [2], [3], [4], [5], [6] could be applicable to 5G signals. Moreover, in [6], tracking and acquisition techniques were proposed, analyzed and tested based on the correlator output mathematical model presented in [5]. However, this model was derived by assuming a constant propagation channel over the duration of the correlation (which is performed over one OFDM symbol). Nevertheless, an analysis conducted on the Channel Impulse Response (CIR) of a representative 5G signals propagation channel, QuaDRiGa [7], has shown that the CIR cannot be considered as constant over the duration of the correlation. Moreover, this analysis has also shown that the expected 5G signal processing behavior is different between a time-evolving CIR and a constant one over the duration of an OFDM symbol.

This article aims thus at extending the DVB-T signals ranging module (Delay Lock Loop - DLL) presented in [5] for constant CIR propagation channels to a $5 \mathrm{G}$ signals ranging module (DLL) for time-evolving CIR propagation channels. The key point of this derivation consists in developing an evolved mathematical model for $5 \mathrm{G}$ signals correlator outputs which takes into account the CIR evolution over the duration of one OFDM symbol.

In order to meet this objective, the article is organized in 8 sections in addition to the introduction and conclusion. First, 5G signals structure and a generic communication chain scheme are presented. Second, the architecture of the envisioned DLL based on [6] is presented for an AWGN. Third, the correlation operation in AWGN is presented. Fourth, the correlator output operation is defined, and its mathematical model is developed and validated for a CIR constant over the duration of the correlation. Fifth, the model for a time-evolving CIR is derived. Sixth, the correlator output mathematical model is developed and validated for a time-evolving CIR. Seventh, the $5 \mathrm{G}$ signals compliant propagation channel being adopted, QuaDRiGa, 
[7] is presented. Finally, the code delay tracking error of the derived 5G signal tracking module is provided for an AWGN channel and for the QuaDRiGa propagation channel model.

\section{5G SIGNAL PRESENTATION}

5G systems use OFDM signals. The process to generate an OFDM signal is illustrated in Fig.1. The symbols $\left\{d_{0}^{k}, \ldots, d_{N-1}^{k}\right\}$ are first modulated by applying an inverse Fast Fourier Transform (IFFT). Then the Cycle Prefix (CP) is added creating an OFDM time symbol. At this point the complex envelope signal model (consisting in one OFDM time symbol) can be expressed as in (1).

$$
s^{k}[n]=\sum_{p=0}^{N_{F F T^{-1}}} d_{p}^{k} e^{\frac{i 2 \pi p n}{N_{F F T}}}-N_{C P} \leq n \leq N_{F F T}-1
$$

Where $N_{C P}$ is the number of Cycle Prefix samples, $N_{F F T}$ is the size of the FFT window, $p$ is the subcarrier, $n$ indicates the $n^{\text {th }}$ discrete time epoch, $k$ indicates the $k^{\text {th }}$ OFDM symbol, $d_{p}^{k}$ is the $k^{t h}$ modulated symbol carried by the $p^{\text {th }}$ subcarrier.

The OFDM signal is then transmitted through the propagation channel, which CIR mathematical model is usually expressed as $\alpha^{k}(t)$. The received signal mathematical model can then be expressed by the convolution between the incoming signal and the previously defined CIR propagation channel, where $r^{k}(t)$ expresses the noiseless received signal before the ADC/AGC.

$$
r^{k}(t)=s^{k}(t) * \alpha^{k}(t)
$$

The CP is removed from this received signal, and finally, the signal is demodulated using a Fast Fourier Transform (FFT).

\section{III.ENVISIONED 5G RANGING MODULE}

The main objective of this article is to design a $5 \mathrm{G}$ signal ranging module performing the time-of-arrival estimation thanks to a Delay-Lock Loop (DLL). The aim of the ranging module is thus to estimate the transmitted signal propagation delay $\tau$ between the $5 \mathrm{G}$ emitter and receiver. The selected generic DLL diagram block scheme is illustrated in Fig2, [1], [6]; this scheme is adapted for an AWGN channel. The tracking step provided by Fig. 2 is preceded by an acquisition step. The acquisition process consists in using algorithms such as the Van de Beek algorithm, [9], followed by the matching pursuit, presented in [6], to achieve a rough time and frequency synchronization with the incoming signal. The tracking (fine estimation) of the rough estimated propagation delays obtained during the acquisition phase is then conducted by the DLL.

The DLL makes use of correlation between the incoming signal, $r^{k}[n]$. and a local replica, $L R$, performed on the pilot symbols that are present in the $5 \mathrm{G}$ Synchronization Signals. The CIR mathematical model of an AWGN channel can be expressed as $\alpha^{k}(t)=\alpha \delta(t-\tau)$, where $\alpha$ is the complex

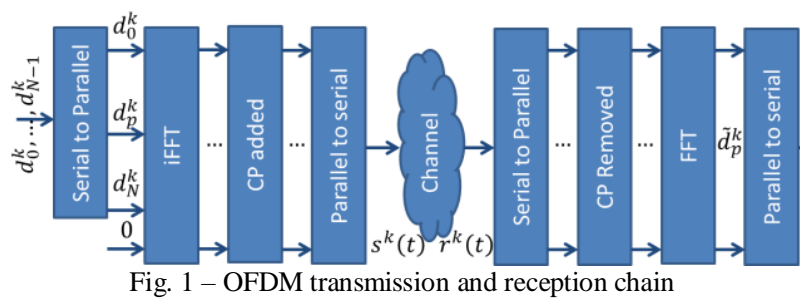

amplitude of the AWGN propagation channel and $\tau$ is the propagation time delay, The noiseless received signal mathematical model can then be derived from the convolution between the incoming signal and this CIR propagation channel as: $r^{k}(t)=s^{k}(t) * \alpha^{k}(t)$. the digital version, $r^{k}[n]$, is provided in (3).

$$
r^{k}[n]=\alpha \sum_{p=0}^{N_{F F T}-1} d_{p}^{k} e^{\frac{i 2 \pi p\left(n-\tau_{n}\right)}{N_{F F T}}}
$$

The propagation delay estimated by the DLL is denoted as $\hat{\tau}$. The correlation between the incoming signal and the local replica is computed at three points spaced by a distance $d$ called the correlator spacing $(d=4$ samples in the following).

- $\hat{\tau}-\frac{d}{2}$ for the early correlator output: $R_{E}\left(\varepsilon_{\tau}\right)$

- $\hat{\tau}$ for the prompt correlator output: $R_{P}\left(\varepsilon_{\tau}\right)$

- $\hat{\tau}+\frac{d}{2}$ for the late correlator output: $R_{L}\left(\varepsilon_{\tau}\right)$

Where $\varepsilon_{\tau}=\tau-\hat{\tau}$ is defined as the propagation delay estimation error. The discriminator output is then computed thanks to these 3 correlator outputs. The discriminator chosen is the Early Minus Late Power (EMLP) defined in (4) and studied in [6]:

$$
D_{E M L P}^{\text {norm }}\left(\varepsilon_{\tau}\right)=\frac{\left|R_{E}\left(\varepsilon_{\tau}\right)\right|^{2}-\left|R_{L}\left(\varepsilon_{\tau}\right)\right|^{2}}{K_{\text {norm }}\left|R_{P}\left(\varepsilon_{\tau}\right)\right|^{2}}
$$

This discriminator is normalized by $K_{\text {norm }}$ such that $D_{E M L P}^{n o r m}\left(\varepsilon_{\tau}\right)=\varepsilon_{\tau}$ for $\varepsilon_{\tau}$ close to 0 , its expression is derived in [6]. The discriminator output is then filtered by a low-pass filter. The new delay estimate is then generated using a DCO (Digitally Controlled Oscillator) and new Early, Prompt and Late correlator outputs are computed. Finally, the correlation operation is the basis of the ranging module as shown in this section. Therefore, the correct mathematical modelling of its output is mandatory in order to develop a ranging module adapted to the targeted propagation channel. This modelling will be the aim of the next sections.

\section{IV.CORRELATION OPERATION DEFINITION}

The correlation operation can be defined for an OFDM symbol from its direct time-domain expression or from the equivalent frequency-domain expression. The schemes of the time-domain and frequency-domain correlation operations are given in Fig.3. In the time domain, the incoming signal is multiplied with the local replica, and then accumulated (integration and dump) to get the correlation result. In the frequency domain, the incoming signal is first demodulated thanks to a FFT operation and then it is multiplied with the FFT of the local replica. Then, an iFFT is performed in order to come back to the time domain. Only the first term of the iFFT output is considered and denominated as "IFFT in 0" in Fig. 3.

Additionally, note that the received signal is also demodulated by applying a FFT as presented in (5) when $\tau$

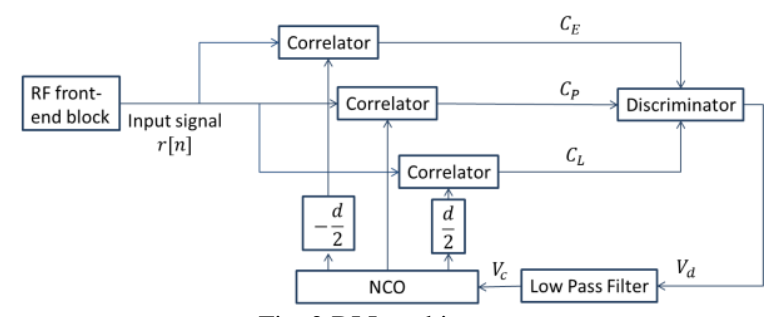

Fig. 2 DLL architecture 


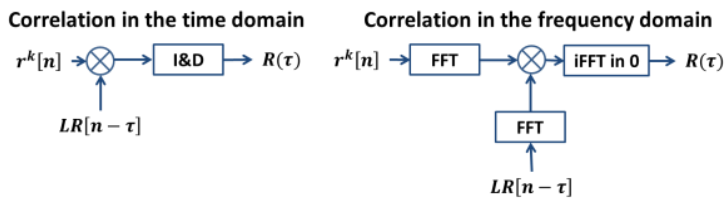

Fig. 3 - Correlation operation

is accurately estimated.

$$
\tilde{d}_{p^{\prime}}^{k}=F F T\left(r^{k}[n]\right)\left[p^{\prime}\right]=\sum_{n=0}^{N_{F F T^{-1}}} r^{k}[n] e^{-i 2 \pi \frac{n p^{\prime}}{N_{F F T}}}
$$

With such expressions it is easier to tune the shifting process in the frequency domain. Therefore, the correlator output mathematical model is derived from this implementation, i.e. the FFT in the frequency domain.

\section{CORRELATOR OUTPUT MATHEMATICAL MODEL FOR A CONSTANT CIR OVER ONE OFDM SYMBOL}

In this section, the correlator output mathematical model is derived for a constant CIR over one OFDM symbol. A more general propagation channel CIR mathematical model is now expressed as follow $\alpha^{k}(t)=$ $\sum_{l=0}^{L-1} \alpha_{l}^{k} \delta\left(t-\tau_{l}\right)$. Where $\alpha_{l}^{k}$ is the complex amplitude of the $k^{t h}$ OFDM time symbol and the $l^{t h}$ path, $\tau_{l}$ is the delay of the $l^{\text {th }}$ multipath, $L$ is the total number of multipath and $l$ is the multipath id. The noiseless received digital signal mathematical model $r^{k}[n]$ is provided in (6).

$$
r^{k}[n]=\sum_{l=0}^{L-1} \alpha_{l}^{k} \sum_{p=0}^{N_{F F T}-1} d_{p}^{k} e^{\frac{i 2 \pi p\left(n-\tilde{\tau}_{l}\right)}{N_{F F T}}}
$$

Where $\tilde{\tau}_{l}=\tau_{l} / T_{s}$ is the normalization of the continuous parameter by the sampling time $T_{S}$.

The correlation is performed on pilots only; the general correlation function expression is defined in [6] as:

$$
R(\tau)=\frac{1}{N_{p}} \sum_{p \in P} \tilde{d}_{p}^{k} L R_{p}^{k *}
$$

Where $L R_{p}^{k}=p_{p}^{k} e^{-\frac{2 i \pi p}{N_{F F T}} \tau}, p_{p}^{k}$ is the pilot symbol localized in the $p^{t h}$ subcarrier on the $k^{\text {th }}$ symbol. $N_{p}$ is the number of pilot symbols and $P$ is the set of pilots. In order to have a generic formula, the set of pilots $P$ can be defined as: $P=\gamma p^{\prime}+\beta$. Where $\gamma$ is the period of repetition of the pilots in the OFDM symbol, $p^{\prime} \in\left[0 \ldots N_{P}-1\right]$ and $\beta$ is the subcarrier index of the first pilot in the symbol. Applying this equation, the final expression of the correlator output is given in (8) with $\varepsilon_{\tau_{l}}=\left(\tau-\tau_{l}\right)$.

$R\left(\varepsilon_{\tau}\right)=\left\{\begin{array}{cc}\sum_{l=0}^{L-1} \frac{\alpha_{l}^{k} e^{\frac{i \pi\left(2 \beta+\gamma\left(N_{p}-1\right)\right) \varepsilon_{\tau_{l}}}{N_{F F T}}} \frac{\sin \left(\frac{\pi \gamma \varepsilon_{\tau_{l}} N_{P}}{N_{F F T}}\right)}{N_{F F T} N_{P}}}{\sin \left(\frac{\pi \gamma \varepsilon_{l}}{N_{F F T}}\right)} & \varepsilon_{\tau_{l}} \neq 0 \\ \frac{1}{N_{F F T}} \sum_{l=0}^{L-1} \alpha_{l}^{k} & \varepsilon_{\tau_{l}}=0\end{array}\right.$

This formula has been validated using $5 \mathrm{G}$ pilots. After deep study of the 3GPP standard, [8], only one set of pilots can be used since its scheme is the only one fixed and predictable: the 4-OFDM-symbol-long Synchronization Signal Physical Broadcast CHannel (SSPBCH). The detection and decoding of this block allow the user to achieve downlink synchronization in time/frequency domain and to obtain $5 \mathrm{G}$ signal characteristics such as the cell identity or the bandwidth; information required to perform the communication with the network. More specifically, in the following, the focus is done on the second (and fourth) symbol which are composed of 1 Demodulation Reference Signal (DMRS) each 4 subcarriers over 240 subcarriers: $N_{p}=60, \gamma=4, \beta=0$ in the following.

\section{VI.MODEL OF A TIME-EVOLVING CIR}

The previously developed model assumes that the propagation channel model is constant over the duration of the OFDM symbol. In this section, the consequences of a non-constant propagation channel on the correlation function are presented.

For a time-evolving propagation channel model, the CIR is modelled as:

$$
\alpha^{k}(t, \tau)=\sum_{l=0}^{L-1} \alpha_{l}^{k}(t, \tau)
$$

The CIR, $\alpha_{l}^{k}(t, \tau)$, for a given received multipath can be expressed as follow:

$$
\alpha^{k}(t, \tau)=\sum_{l=0}^{L-1} \alpha_{l}^{k}(t) \cdot \delta\left(\tau-\tau_{l}\right)
$$

Where $\tau$ is a mathematical variable used for the convolution, $t$ is used to model the complex amplitude evolution of each multipath and $\alpha_{l}^{k}(t)$ is the evolving complex amplitude of the $k^{\text {th }}$ OFDM symbol and the $l^{\text {th }}$ multipath. Then, the received signal for the $l^{\text {th }}$ multipath is calculated as $r(t)=\left.\alpha_{l}^{k}(t) \cdot \delta\left(\tau-\tau_{l}\right) * s(\tau)\right|_{\tau=t}=\alpha_{l}^{k}(t) \cdot s\left(t-\tau_{l}\right)$

Assuming for simplification purposes $\tau_{l}=0$, the received signal Fourier transform is equal to:

$$
R(f)=\mathrm{A}_{l}^{k}(f) * S(f)
$$

Where $\mathrm{A}_{l}^{k}(f)=F T\left[\alpha_{l}^{k}(t)\right]$. If the propagation channel is constant over the duration of the correlation, $\alpha_{l}^{k}(t)=\alpha_{l}^{k}$, $A_{l}^{k}(f)$ is a periodic Dirac function. However, if the channel evolves, $\alpha_{l}^{k}(t) \neq \alpha_{l}^{k}$, then the convolution in the frequency domain will create a spectral broadening effect. This spectral broadening implies the loss of the orthogonality between the subcarriers of the OFDM signal. This process is illustrated in Fig. 4.

\section{CORRELATOR OUTPUT MATHEMATICAL MODEL} FOR A NON-CONSTANT CIR OVER ONE OFDM SYMBOL

\section{A. Correlator output mathematical model derivation}

The previous correlator output formula, equation (8), was derived for a propagation channel constant over the duration of one OFDM symbol. However, in general, 5G signals propagation channels, cannot be considered as constant, e.g. QuaDRiGa [7]. In order to develop a correlator output mathematical model for a time-evolving propagation channel, the method used for a constant CIR was repeated. Equations (13) and (14) show the OFDM demodulated symbol and correlator output mathematical models:

$$
\begin{gathered}
\tilde{d}_{p}^{k}=d_{p}^{k}+\text { Interference } \\
R\left(\varepsilon_{\tau}\right)=R_{\text {useful }}\left(\varepsilon_{\tau}\right)+R_{\text {interf }}\left(\varepsilon_{\tau}\right)
\end{gathered}
$$

It must be noted that the useful term, in (14), is different from the correlator output derived in (8) for a constant channel. Fig.7 can help to understand the impact of the channel on a particular symbol $k$. For a constant channel, only one coefficient is considered for each path $l$ for each symbol $k, \alpha_{l}^{k}$ (violet curve); while for an evolving channel, the impact of the $l$-th path on symbol $k$ is modelled by the

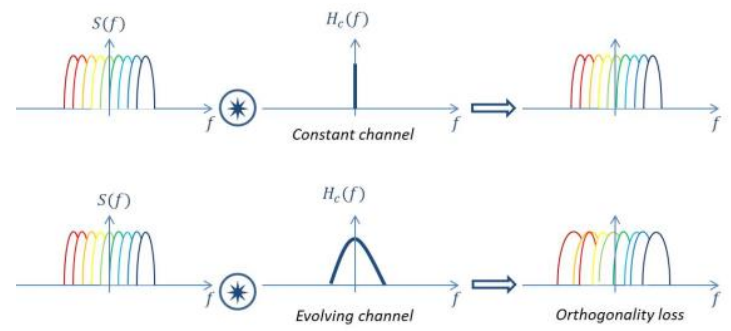

Fig. 4 Impact of a non-constant propagation channel 
$\operatorname{sum} \sum_{n=0}^{N_{F F T^{-1}}} \alpha_{l}^{k}(n)$, the values $\alpha_{l}^{k}(n)$ will be constant over period shorter than the OFDM symbol, and will therefore depend on the chosen CIR sampling rate. In Fig. 7, 3 different sampling rates are illustrated: 10, 20 and $100 \mathrm{CIR}$ sps.

The statistics of the two terms, useful and interference, of the OFDM demodulated symbols as well as the correlator output mathematical model have been analyzed for QuaDRiGa channel (presented in next section). The study proves the negligibility of the interference term with respect to the useful term for this channel. Mathematical demonstrations are not provided here, nevertheless, in Fig.5, an illustration is provided as a justification. Monte-Carlo simulations have been used to assess the amplitude of the useful and interference term of the correlation function for a CIR sampling rate of 100 CIR samples per symbol. On the left is presented the correlation main peak, on the middle the useful (up) and interference (bottom) terms and on the right, the useful and interference terms contributions; which are equal to $100.0048 \%$ and $0.0789 \%$ of the total correlation function respectively. The contribution is computed using: $\left|R_{\text {useful }}\left(\varepsilon_{\tau}\right)\right| /\left|R_{\text {useful }}\left(\varepsilon_{\tau}\right)+R_{\text {interf }}\left(\varepsilon_{\tau}\right)\right| \cdot 100 \quad$ and $\left|R_{\text {interf }}\left(\varepsilon_{\tau}\right)\right| /\left|R_{\text {useful }}\left(\varepsilon_{\tau}\right)+R_{\text {interf }}\left(\varepsilon_{\tau}\right)\right| \cdot 100$ respectively; this permits to understand that the interference term can be destructive, making the useful contribution higher than $100 \%$. Therefore, the final model adopted is limited to $R_{\text {useful }}\left(\varepsilon_{\tau}\right)$ which is defined in (15):

$$
\begin{aligned}
& R\left(\varepsilon_{\tau}\right) \approx R_{\text {useful }}\left(\varepsilon_{\tau}\right)= \\
& \left\{\begin{array}{cc}
\sum_{l=0}^{L-1} \frac{A_{l}^{k}(0)}{N_{F F T} N_{P}} e^{\frac{i \pi \varepsilon_{\tau}\left(2 \beta+\gamma\left(N_{P}-1\right)\right.}{N_{F F T}} \frac{\sin \left(\frac{\pi \gamma \varepsilon_{\tau} N_{P}}{N_{F F T}}\right)}{\sin \left(\begin{array}{c}
\pi \varepsilon_{\tau} \\
N_{F F T}
\end{array}\right)}} & \varepsilon_{\tau} \neq 0 \\
\frac{1}{N_{F F T}} \sum_{l=0}^{L-1} A_{l}^{k}(0) & \varepsilon_{\tau}=0
\end{array}\right.
\end{aligned}
$$

\section{B. Simplified OFDM correlator output mathematical} models for a non-constant CIR over one OFDM symbol

The correlator output mathematical model contains the $\operatorname{sum} A_{l}^{k}(0)=\sum_{n=0}^{N_{F F T}-1} \alpha_{l}^{k}(n)$ which contains the impact of the evolution of the propagation channel over one symbol for the $l^{\text {th }}$ multipath. The objective of this section is to simplify this term in order to further simplify the complete correlator output mathematical model. A potential application of this simplified model would be the implementation of a simulator; where the computational burden is an important factor that must not be forgotten.

In order to simplify $A_{l}^{k}(0)$, the following hypotheses are considered:

- The sum of the complex amplitudes is split in two sums, the sum over the modulus and the sum over the phases:

$\sum_{n=0}^{N_{F F T}-1} \alpha_{l}^{k}(n) \approx\left(\sum_{n=0}^{N_{F F T}-1}\left|\alpha_{l}^{k}(n)\right|\right)\left(\frac{\sum_{n=0}^{N_{F F T^{-1}}} e^{i \theta(n)}}{N_{F F T}}\right)$

- The sum over the modulus is replaced by the mean value of the first modulus of the channel impulse response at the instants of the symbols $k$ and $k+1$ :

$\sum_{\mathrm{n}=0}^{\mathrm{N}_{\mathrm{FFT}}-1}\left|\alpha_{\mathrm{l}}^{\mathrm{k}}(\mathrm{n})\right| \approx\left(\left|\alpha_{1}^{\mathrm{k}}(0)\right|+\left|\alpha_{\mathrm{l}}^{\mathrm{k}+1}(0)\right|\right) \mathrm{N}_{\mathrm{FFT}} / 2$

- The phase variation is assumed linear over one symbol (this assumption has been verified through simulations): $\theta(n)=\theta_{0}^{k}+2 \pi \delta_{f} \cdot n$. Where $\theta_{0}^{k}$ is the phase of the

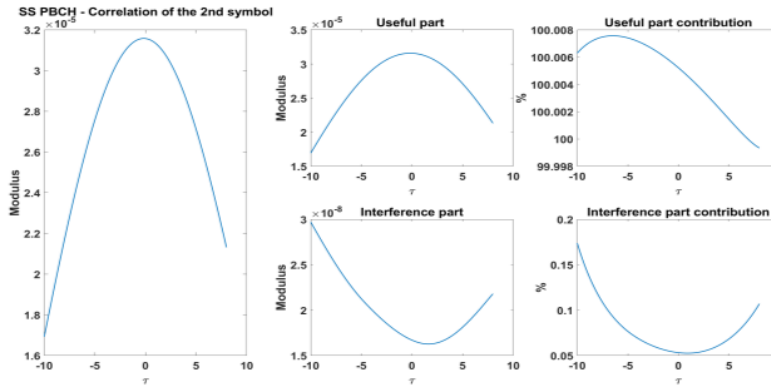

Fig 5. Negligibility of the interference term

first propagation channel sample applied to the $k^{\text {th }}$ OFDM symbol and $\delta_{f}=\left(\theta_{0}^{k}-\theta_{0}^{k+1}\right) /\left(2 \pi N_{F F T}\right)$ is the normalized Doppler frequency.

This simplification would allow generating only 1 CIR sample per OFDM symbols instead of $N_{C I R}$ CIR samples, which would greatly reduce the computation time of propagation channel models, e.g. QuaDRiGa. $N_{C I R}$ represents the required number of CIR samples per OFDM symbol to obtain a propagation channel model equivalent to a time-continuous channel.

Finally, the channel contribution term can be simplified as presented in (18).

$$
A_{l}^{k}(0) \approx \frac{\left|\alpha_{l}^{k}(0)\right|+\left|\alpha_{l}^{k+1}(0)\right|}{2} e^{i\left(\theta_{0}+\pi \delta_{f} \cdot\left(N_{F F T}-1\right)\right)} \frac{\sin \left(\pi \delta_{f} N_{F F T}\right)}{\sin \left(\pi \delta_{f}\right)}
$$

\section{QUADRIGA: A 5G PROPAGATION CHANNEL}

\section{A. Presentation of the channel}

In this article, the propagation channel selected to validate the correlator output mathematical models developed in the previous sections is the QuaDRiGa propagation channel, [7].

QuaDRiGa allows choosing between different scenarios representative of $5 \mathrm{G}$ signals propagation channel models. In this article, among the proposed configurations "3GPP_38.901_UMi_LOS" has been selected. This scenario models typical terrestrial pico-base stations deployed below rooftop in densely populated urban areas covering carrier frequencies from $500 \mathrm{MHz}$ to $100 \mathrm{GHz}$. The Line-Of-Sight (LOS) scenario is a favorable case; the LOS signal is received with significant higher power than multipath. Picobase stations have typically a 4-to-200m-radius-size; thus, the trajectory simulated is a few meter-long arc of a $100 \mathrm{~m}-$ radius circle. Finally, regarding the 5G signal characteristics, the carrier frequency used is set to $6 \mathrm{GHz}$ and the subcarrier is $15 \mathrm{kHz}$.

\section{B. Time-evolving CIR correlator output validation using QuaDRiGa}

In order to validate the different correlator output mathematical models presented in section VII, the following method is applied. First, the correlation is computed over 2000 symbols for four cases:

1. the correlation operation using a sampling interval of 100 CIR samples per OFDM symbols (the reference),

2. the correlation operation using a sampling interval of 10 CIR samples per OFDM symbols,

3. the correlation using a sampling interval of $10 \mathrm{CIR}$ samples per OFDM symbols using (15), and

4. the correlation using (18), using only 1 CIR sample per OFDM symbol. 

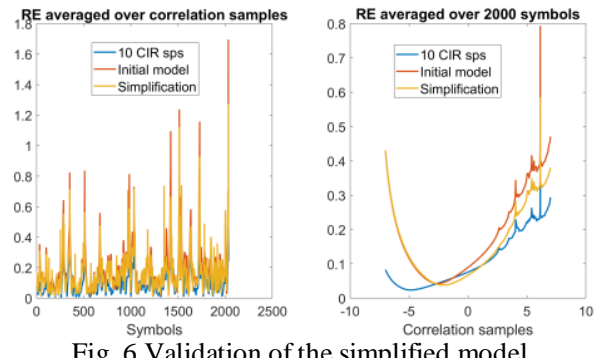

Fig. 6 Validation of the simplified model

The correlation operation refers to the correlation obtain after performing the complete OFDM transmission chain of Fig. 1 (generation of OFDM symbol, modulation, CP addition, channel degradation, $\mathrm{CP}$ removal, demodulation, correlation). Second, the last three correlation output values are compared to the reference in terms of relative evolution (RE). Results are averaged with respect to the main correlation peak (right of Fig.6) and with respect to the 2000 symbols (left of Fig.6). Results are summarized in Table. 1

TABLE 1 - RELATIVE EVOLUTION MEAN AND STANDARD DEVIATION

\begin{tabular}{|c|c|c|c|}
\hline Model & $10 \mathrm{CIR}$ & Initial & Simplification \\
\hline$\mu$ & $0.1267 \%$ & $0.1379 \%$ & $0.1016 \%$ \\
\hline$\sigma$ & $0.1178 \%$ & $0.1237 \%$ & $0.0866 \%$ \\
\hline
\end{tabular}

These results permit to validate the usage of the simplified correlator outputs mathematical model. Results obtained with the simplified model are even better than the two previous one. In order to understand this result, see Fig 7. This figure illustrates the method used to model the time evolution of the propagation channel over one OFDM symbol. Supposing that an OFDM symbol is composed of $\mathrm{N}$ signal samples and that X CIRs are applied, then over N/X samples, the applied channel coefficients are constant. It can be seen that the first complex amplitude of symbol $k+1$ is closer to the last complex amplitude applied to symbol $k$ with the reference than the last complex amplitude of symbol $k$ obtained with $10 \mathrm{CIR}$. It means that the complex amplitudes considered in the simplified model are closer to the reference, which explained the results.

\section{APPLICATION OF THE CORRELATOR OUTPUT MODEL TO THE CODE DELAY TRACKING OF A 5G SIGNAL}

The correlator output mathematical model developed in the previous sections is applied to the code delay tracking of a $5 \mathrm{G}$ signal-type, defined in section III. The performances of the DLL are provided for 3 cases:

1. an AWGN channel,

2. a multipath channel and

3. a LOS channel.

The tracking performance is analyzed by calculating the variance of the estimated delay. In Fig. 8, the standard deviation of the tracking error estimate is provided in

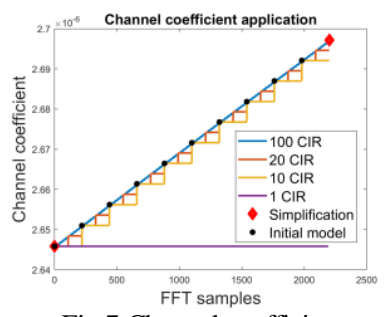

Fig.7 Channel coefficient application

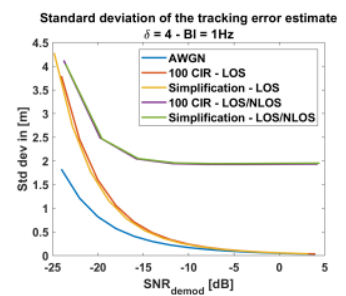

Fig. 8 Standard deviation of the tracking error estimate function of the SNR for an AWGN, for a multipath channel and for a channel composed of only a LOS signal. According to this study, the $5 \mathrm{G}$ ranging performance can provide sub-meter accuracy $(3 \sigma)$ for SNR as low as OdB for the AWGN; this provides the optimal performances that can be reached. In red and yellow, the standard deviation is provided for a channel composed of only one LOS path; these curves are computed using the reference correlation (with 100 CIR sampling rate) and the simplified model from (18) respectively. In violet and green, a multipath channel is used; these curves are computed using the reference correlation (with $100 \mathrm{CIR}$ sampling rate) and the simplified model from (18) respectively. Two conclusions can be made from this figure. First, the validity of the simplified model is proved. Second, due to the multipath, there is a threshold from which the precision of the DLL cannot be improved.

\section{CONCLUSION}

This article has proposed a $5 \mathrm{G}$ ranging module (DLL), using an EMPL discriminator, for non-constant over an OFDM symbol duration CIR propagation channels. This proposal has required the derivation of OFDM-type signal processing mathematical models for a time-evolving CIR of the different stages of a navigation receiver: OFDM demodulated symbols and correlator outputs.

The developed correlator output mathematical model was applied to a $5 \mathrm{G}$ pilots, using a realistic channel model (QuaDRiGa). The development of these mathematical models has been validated by comparing the tracking error standard deviation using the correlation model using 100 CIR sps and the simplified correlation model. These simulations show that the same performance is observed, even though the sampling of the channel model has been reduced by a factor 100 .

Future work will now use the simplified correlator output model to implement a realistic simulator that will allow the evaluation of the $5 \mathrm{G}$ positioning performance and the development of innovative techniques to optimize the processing of such signal for positioning. The simplified correlator model could also be applied on the promising $5 \mathrm{G}$ mm-wave signals, which would have a different emitter network topology and signal characteristics, and where the channel characteristics evolve even more rapidly within an OFDM symbol duration.

\section{REFERENCES}

[1] K.Shamaei, J.Khalife, Z.M. Kassas, "Exploiting LTE Signals for Navigation: Theory to Implementation", 2018

[2] C.Mensing, S.Plass, A.Dammann, "Synchronization algorithms for positioning with OFDM communciations signals", 2007

[3] C.Mensing, A. Dammann, "Positioning with OFDM based communications systems and GNSS in critical scenarios", 2008

[4] E.Staudinger, "A generic OFDM based TDoA positioning testbed with interference mitigation for subsample delay estimation", 2011

[5] P.Thevenon, D.Serant, O.Julien, C.Macabiau, "Positioning using mobile TV based on DVB-SH standard", 2014

[6] D.Serant, P.Thevenon, O.Julien, C.Macabiau, "Development and validation of an OFDM/DVB-T sensor for positioning", 2010

[7] S. Jaeckel, L. Raschkowski, "QuaDRiGa: A 3-D Multicell Channel Model with Time Evolution for Enabling Virtual Field Trials," 2014.

[8] 3GPP, "TS 38.211 Physical channels and modulation".

[9] J-J Van de Beek, M. sandell, P.O. Börjesson, "ML estimation of Time and Frequency Offset in OFDM Systems", 1997 